Check for updates

Cite this: RSC Adv., 2018, 8, 36987

Received 1st May 2018

Accepted 27th September 2018

DOI: $10.1039 / c 8 r a 03724 h$

rsc.li/rsc-advances

\section{An AC electrothermal self-circulating system with a minimalist process to construct a biomimetic liver lobule model for drug testing $\dagger$}

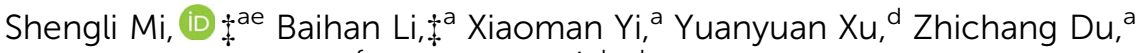 \\ Shuaitao Yang, ${ }^{a}$ Wei Li ${ }^{f}$ and Wei Sun ${ }^{\star a b c d}$
}

\begin{abstract}
Liver-on-chip, due to its precision and low cost for constructing in vitro models, has tremendous potential for drug toxicity testing and pathological studies. By applying APAP (acetaminophen) treatment of different concentrations, a dynamic self-circulating liver lobule model for drug testing was proven useful for emulating the human physiological system. However, the demand for a dynamic system of on-chip organs is difficult to fulfil due to the relatively cumbersome fabrication processes. In this paper, the design and fabrication of an AC electrothermal self-circulating system combined with a 3D biomimetic liver lobule is described. The system was fabricated using a low-cost ITO laser etching process within a few seconds. A large number of interdigital electrodes were arranged in a limited space to increase the fluid flow-driven efficiency. The liver lobule consists of two parts, a hepatocyte cell-laden layer and an endothelial layer, which exhibit a sandwich radial shaped pattern that is more bionic in structure and function. By evaluating the velocity and temperature in the self-circulating system at various voltages and frequencies, we obtained a set of reliable input parameters to provide an adequate supply of culture fluid without cell damage. The metabolism of the liver lobule in dynamic culture and static culture was compared based on cell viability, albumin secretion and urea synthesis.
\end{abstract}

\section{Introduction}

Due to the high cost of pharmaceutical development to conduct toxicity testing on live animals and human organs and the need for basic physiological and pathological research, a substantial amount of work has been devoted towards constructing in vitro tissue models to realize part of the cellular microenvironment and tissue function. ${ }^{1}$ From early microfluidic chips for protein fragment testing and multicellular co-culturing to microtissue or micro-organ systems, such as co-culture systems of hepatocytes and liver endothelial cells, the existing models have been extended to MPS (microphysiological systems), and the future trend is to build so-called human-on-chip devices for various pharmacokinetic studies. ${ }^{2-6}$ However, there is currently no

${ }^{a}$ Graduate School at Shenzhen, Tsinghua University, Shenzhen, P. R. China. E-mail: weisun@mail.tsinghua.edu.cn; Fax: +8675526036356; Tel: +8675526036329

${ }^{b}$ Department of Mechanical Engineering and Mechanics, Tsinghua University, Beijing, China

${ }^{c}$ Department of Mechanical Engineering, Drexel University, Philadelphia, PA, USA

${ }^{d}$ Tsinghua-Berkeley Shenzhen Institute, Shenzhen, P. R. China

${ }^{e}$ Open FIESTA Center, Tsinghua University, Shenzhen 518055, PR China

${ }^{f}$ Department of Mechanical Engineering, The University of Texas at Austin, Austin, Texas 78712, USA

$\dagger$ Electronic supplementary information (ESI) available. See DOI: 10.1039/c8ra03724h

\$ These authors contributed equally to this work. closed-loop liver chip that simulates the in vivo dynamic circulation and achieves the control of flow rates in micro-tissues or micro-organs. ${ }^{7}$ Hepatic lobule is the smallest unit of liver, and it bears multiple functions of human body including detoxification, dynamic metabolism and immune response ${ }^{8}$ and the establishment of an entire microphysiological circulatory system and the biomimetic construction of a hepatic lobule model are key to the realization of a model of liver metabolism and drug testing.

Recent advances in tissue engineering and microfabrication technologies, which allow the manipulation of various cell types to grow rapidly in vitro, have provided an unprecedented opportunity to fulfil this purpose. ${ }^{9-11}$ To this end, some remarkable work has focused on reconstructing 3D multilayer structures, ${ }^{12-14}$ especially a radial star-shaped structure, which contains multiple liver tissue cells to mimic the functional unit of liver lobule. ${ }^{15}$ For example, to create different phase boundaries to generate sinusoid-like models, a biomimetic artificial hepatic sinusoid with a microfluidic endothelial barrier was built with the liver acinus-like function for mass transportation., ${ }^{4,16}$ Additionally the use of a porous membrane, such as PET $^{17,18}$ or silicon nitride ${ }^{19}$ in place of barriers can effectively reduce the boundary inhomogeneities resulting from the barrier structure. Although these in vitro approaches imitating the structure of hepatic cord could be applied for the study for toxicity testing, they do not constitute the actual morphology of 
hepatic lobule and the capability for mass transportation would be degraded by applying porous membrane. Recently, one noteworthy approach applying the principle of dielectrophoresis with two different sets of electrodes could trap HepG2 cells and vein endothelial cells respectively to form a radial shaped hepatic lobule. $^{20}$ In another approach, a DLP (digital light process) based 3D bioprinting system was applied to develop a 3D hydrogel-based triculture model. ${ }^{21}$ However, a hydrogel with cells can only be manufactured in a thin layer using the above methods, and large-scale cell-hydrogel assemblies would be achieved by stacking cell-embedded mesh-like hydrogel sheets and curing layer by layer to form a sandwich shaped radial structure. ${ }^{22}$ However, the curing method using alginate with calcium chloride does not provide as good an environment for free cells growth as tail collagen. As for dynamic culture, an extensible liver lobule microphysiological system consisting of an integrated network of hexagonal chambers realized the distribution of blood vessels and the state of blood flow. ${ }^{23}$ Another approach using a $\mathrm{P} \mu \mathrm{s}$ (pneumatic microvalve system) to actuate two independent cell suspension flows for cell capturing arrays to construct a radial hepatic cord-like network showed a more obvious physiological metabolites of the liver and a faster drug reaction rate. ${ }^{24}$ However, there are some limitation of the above two in that the syringe pumps or the relatively large external devices, such as gas cylinders cannot supply liquid to many receptors in a limited space. In our previous study, we reported the research of constructing a biomimetic liver sinusoid by taking advantage of laminar flow and analysing the metabolic products by the effects of drug toxicity. ${ }^{25}$ Although the simulation of blood flow in vitro was performed by fluid gravity and did not need any other devices to pump it, we could not achieve a relatively stable and controllable flow rate.

Over the past decades, with the maturity of AC electrothermal theory, chips integrated with AC electrodes have emerged to be driven functionally. ${ }^{26,27}$ For example, an AC electrothermal circulatory pumping chip was manufactured to generate a sufficient fluid rate without any harm to cell culture. ${ }^{28}$ However, the electrodes were made by patterning different thicknesses of metal layers, such as gold and platinum on the silicon substrate. The process of depositing electrodes requires a relatively long time and is a relatively difficult manufacturing process. ${ }^{29,30}$ Based on the previous study, we report the design and manufacture of a self-circulating microsystem which mainly consists of two parts: the pumping area and liver lobule model area. The pumping area is driven by AC electrothermal electrodes, which are fabricated easily to enhance the pumping efficiency. We use a laser marking machine to photo-etch the ITO glass surface and produce high density electrodes. The processing time is reduced to only several seconds. The lobule area is a two-layer structure upon which the upper radial shaped layer of HUVECs grows, and hepatocytes HepG2 grows on the lower layer directly with no porous membrane. This design helps reduce the involvement of non-biological materials. Through simulation of the fluid velocity and temperature of the chip and measurement of the main flow velocity and overall temperature, we obtain an AC electrothermal actuating system that simulates blood flow and bionic interstitial flow. The cell morphology and viability under dynamic culture were characterized, and a toxicity test and the expression of liver-specific functions, such as protein secretion and urea synthesis were analysed.

\section{Theory}

When there is an uneven electric field applied in a high ion concentration medium, the electric field induced Joule heating would generate a locally inhomogeneous thermal field in the microfluidic flow channel. We qualify the thermal field as the temperature gradient, $\nabla T$. Subsequently, some properties of the fluid with a close relationship with the temperature gradient (i.e., permittivity and conductivity) would vary according to the temperature change. Therefore, induced free charges shuttles through the nonuniform electric field. The impetus determined by these nonuniform electric fields would actuate the fluid to move along one direction. The phenomenon of AC electrothermal is shown in Fig. 1A.

\subsection{Electrostatics}

The electric potential $\mathrm{V}$ is determined by the Laplace equation: ${ }^{\mathbf{1}}$

$$
\nabla^{2} V=0
$$

The magnitude of the electric field applied through the media, $E$ is determined by the AC electric field $V:^{32}$

$$
E=-\nabla V
$$

\subsection{Heat transfer}

The power density turned into Joule heating from the applied electric fields is given by: ${ }^{33}$

$$
W=\sigma E^{2}
$$

where $\sigma$ is the electrical conductivity.

We can obtain the relationship between the electric-field and Joule heating from the energy balance equation ${ }^{34}$

$$
\rho_{\mathrm{m}} c_{\mathrm{p}} \mathbf{u} \cdot \nabla T+\rho_{\mathrm{m}} c_{\mathrm{p}} \frac{\partial T}{\partial t}=k \nabla^{2} T+\sigma E^{2}
$$

Here, $\rho_{\mathrm{m}}$ is the density, $c_{\mathrm{p}}$ is the specific heat, and $k$ is the thermal conductivity of the medium.

Because the AC temperature equilibrium is quickly reached when the AC current is working and the effect of fluid flow on the temperature distribution can be neglected, the equation can be written as: ${ }^{34}$

$$
k \nabla^{2} T+\sigma E^{2}=0
$$

If the temperature increases very little, a linear approximation can represent the temperature dependent parameters $\sigma$ and $\varepsilon .^{35}$ 
A

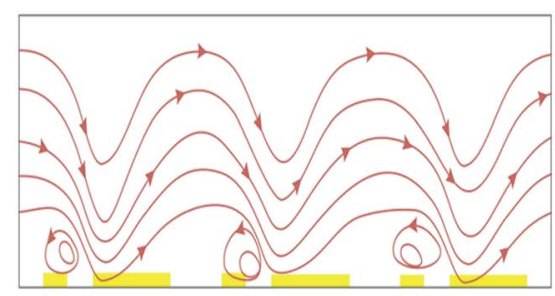

$\mathrm{B}$

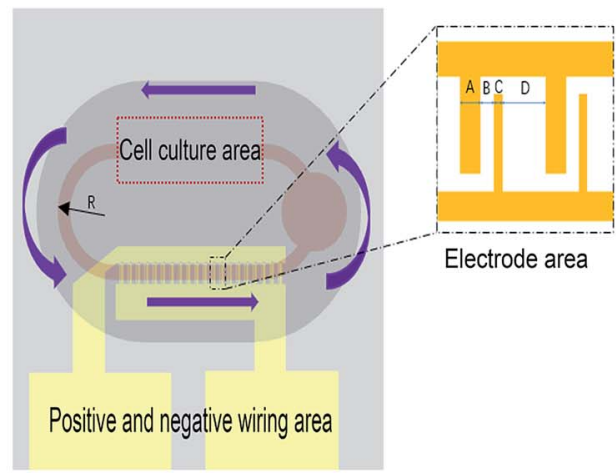

C
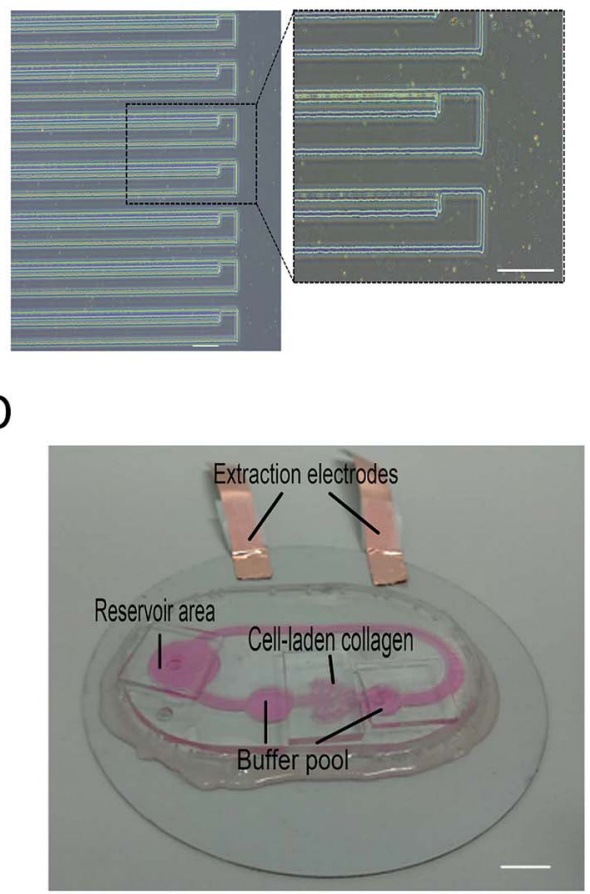

Fig. 1 Design of the self-circulating system. (A) Schematic diagram of the electrothermal conversion pumping liquid medium on AC power (the red represents the fluid flow curve, and the yellow represents the electrode). (B) Schematic diagram of the self-circulating system (the purple arrow represents the liquid flow direction). (C) Fabricated microelectrode array, 20/20/100/150 $\mu \mathrm{m}$ (narrow electrode width/gap/wide electrode width/space between electrode pairs, and scale bar, left $=100 \mu \mathrm{m}$, right $=100 \mu \mathrm{m}$ ). (D) Photo of the self-circulating system of the integrated liver-lobule model, and scale bar $=5 \mathrm{~mm}$.

$$
\begin{gathered}
\varepsilon(T)=\varepsilon\left(T_{0}\right)\left(1+\alpha\left(T-T_{0}\right)\right) \\
\sigma(T)=\sigma\left(T_{0}\right)\left(1+\beta\left(T-T_{0}\right)\right)
\end{gathered}
$$

Here, $\varepsilon$ is the electrical conductivity of the solution.

For an aqueous solution, the approximation of $\alpha$ and $\beta$ can be expressed as: $:^{35}$

$$
\begin{gathered}
\alpha=\left.\frac{1}{\varepsilon\left(T_{0}\right)} \frac{\partial \varepsilon}{\partial T}\right|_{T_{0}}=-0.004 / \mathrm{K} \\
\beta=\left.\frac{1}{\sigma\left(T_{0}\right)} \frac{\partial \sigma}{\partial T}\right|_{T_{0}}=0.02 / \mathrm{K}
\end{gathered}
$$

where $T_{0}$ represents the ambient temperature.

\section{Fluid flow}

We use the Navier-Stokes equation and the equation of continuity to illustrate the incompressible fluid that has low Reynolds number. ${ }^{36-38}$

$$
-\nabla p+\eta \nabla^{2} \mathbf{u}+\left\langle\boldsymbol{F}_{\boldsymbol{E} \boldsymbol{T}}\right\rangle=0, \nabla \cdot \mathbf{u}=0
$$

Here $p$ is the pressure, $\eta$ is dynamic viscosity and $\mathbf{u}$ represents the velocity vector.

The time average bulk forces that ACET generated can be written as: ${ }^{39}$

$$
\left\langle\boldsymbol{F}_{\boldsymbol{E} \boldsymbol{T}}\right\rangle=\frac{1}{2} \frac{\varepsilon(\alpha-\beta)}{1+(\omega \tau)^{2}}(\nabla T \cdot E) E-\frac{1}{4} \varepsilon \alpha|E|^{2} \cdot \nabla T
$$

where $\omega$ is the angular frequency of the AC electric field and $\tau=$ $\sigma\left(T_{0}\right) / \varepsilon\left(T_{0}\right)$ is the charge relaxation time.

\section{Materials and methods}

\subsection{Design and manufacture of AC electrothermal self- circulating system}

Fig. 1B shows the design of the self-circulating chip. The selfcirculating system consists of three layers, the electrode substrate, the circular flow channel and the upper liver plate, which form a sandwich structure. The following section explains the details of the self-circulating chip and how it is more easily fabricated and more inexpensive than previously reported designs.

4.1.1 Electrode substrate. Compared with the previous fabrication using photolithography with several steps, and the process of wet etching, which is time-consuming and not easily controlled, ${ }^{35}$ we selected a laser marking machine (HANS LASER, 31S20A, Han's Laser Technology Industry Group Co., Ltd) with an accuracy of $20 \mu \mathrm{m}$ to scan the ITO glass $(10 \Omega, 100$ $\times 0.5 \mathrm{~mm}$ ). First, the ITO glass need to be ultrasonically washed with acetone and absolute alcohol and deionized for $10 \mathrm{~min}$. Next, we input the electrode patterning designed with AutoCAD software (Autodesk 2017) into the laser marking machine and the machine is set to a scanning speed is set of $200 \mathrm{~mm} \mathrm{~s}^{-1}$ and 
A

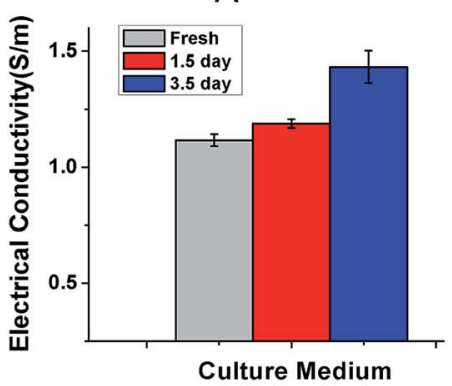

B

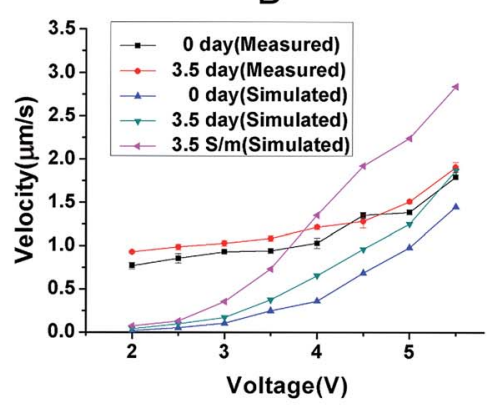

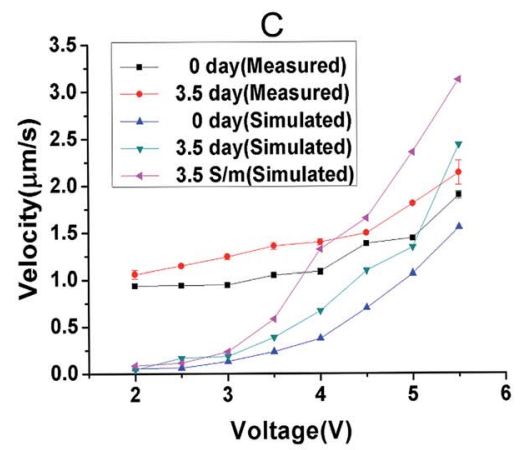

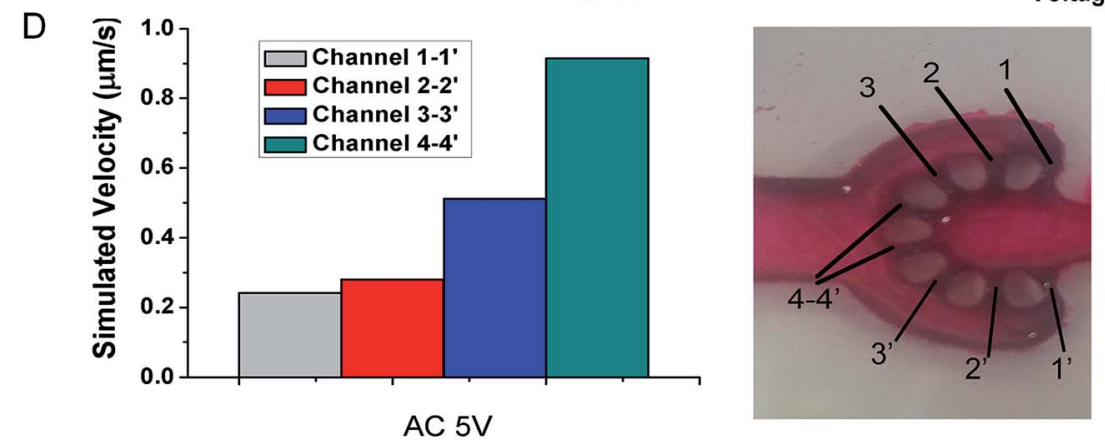

AC 5V

Fig. 2 Evaluation of the self-circulating system. (A) Electrical conductivity tests of the culture medium at 0, 1.5, and 3.5 d. (B) Simulation and measured results of the flow velocities of medium with different electrical conductivities at $1 \mathrm{MHz}$. (C) Simulation and measured results of the flow velocities of medium with different electrical conductivities at $10 \mathrm{MHz}$. (D) Simulation of the flow rate in diverging channels at $V_{p}=5 \mathrm{~V}, \sigma=$ $1.12 \mathrm{~S} \mathrm{~m}^{-1}, f=10 \mathrm{MHz}$, and scale bar $=1.5 \mathrm{~mm}$.

the current is set at $4.5 \mathrm{~A}$. The electrodes fabricated here are 20 $\mu \mathrm{m} / 100 \mu \mathrm{m}$ wide and $4 \mathrm{~mm}$ long and the gap separation between the in-pair and pair-pair are $20 \mu \mathrm{m}$ and $150 \mu \mathrm{m}$, respectively. Finally, the previous cleaning procedure is repeated and determine whether there are any defects under microscope.

4.1.2 Circulating flow channel. We used a DLP (digital light processing) 3D printer to fabricate a ring mould, a linear and radial shaped area and three rounded areas that were located along a closed oval circulating mould. We designed different heights of the mould to form radial area. The width of the branches of the radial area is $700 \mu \mathrm{m}$, and the primary channel is $3 \mathrm{~mm}$. We put the oval circulating mould on a silicon wafer. PDMS (polydimethylsiloxane) curing agent and elastomer base were mixed at proportion of $1: 10$ to pour on the mould. A slightly larger oval polymethylmethacrylate (PMMA) sheet (1.5 mm thick) with two exhaust vents covered the mould. After degassing in a vacuum drying oven and curing at $85{ }^{\circ} \mathrm{C}$ for $30 \mathrm{~min}$, the circulating flow channel is then drafted.

4.1.3 Liver plate and the combination. Using a standard soft lithography method, an oval shaped PDMS (1.5 mm thick) with a circular groove is obtained (diameter $1 \mathrm{~mm}$ and $400 \mu \mathrm{m}$ deep). The positions corresponding to the branch channel exit and solution changing pools are punched with a biopsy puncher. The electrode substrate, the circulating flow channel and the liver plate are treated with oxygen plasma and bonded before and after filling with culture medium and cell-laden collagen. The holes connected with the outside were sealed with several small pieces of a PDMS chip.

\subsection{Formation of 3D biomimetic liver lobule model}

4.2.1 Cell culture and the preparation of cell-laden collagen. The hepatic cell line HepG2 (ATTC) and the endothelial cell line HUVEC (ATTC) were cultured in medium containing $1 \%$ penicillin/streptomycin (P/S) (HyClone), 10\% foetal bovine serum (FBS), and either 90\% DMEM (HyClone, SH30022.01) or 90\% RPMI (HyClone) in a carbon dioxide incubator at $37^{\circ} \mathrm{C}$, respectively. As mentioned in our previous work ${ }^{25}$ the HepG2 cells were resuspended in rat tail Collagen-I (Corning BioCoat, 354236) at a density of $1.5 \times 10^{4} \mathrm{ml}^{-1}$ and the HUVECs were resuspended at a density of $1.5 \times 10^{3} \mathrm{ml}^{-1}$. The medium of the suspension and chip consists of $1 \%$ penicillin/ streptomycin (P/S) (HyClone), 10\% foetal bovine serum (FBS), 40\% DMEM and 40\% RPMI.

4.2.2 Hepatic lobule fabrication. The procedure for the hepatic lobule fabrication is shown in the Fig. 3A. First, we inject $15 \mu \mathrm{l}$ of hepatic cell-laden collagen into the groove of the liver plate and use a cell scraper to smooth the top. Second, after bonding the layers of the liver plate and circulating flow channel, we injected $300 \mu \mathrm{l}$ of HUVECs suspension into the radial shaped area through the small holes, and the medium for dynamic culture was poured into the chip through the large holes for changing the medium. Third, after the holes were sealed with PDMS chips, the entire chip was reversed and placed into an incubator for $12 \mathrm{~h}$ of static culture. The 3D biomimetic hepatic lobule microtissue is shown in the Fig. 3CE. The 3D fluorescence images were taken using laser scanning confocal microscope. 


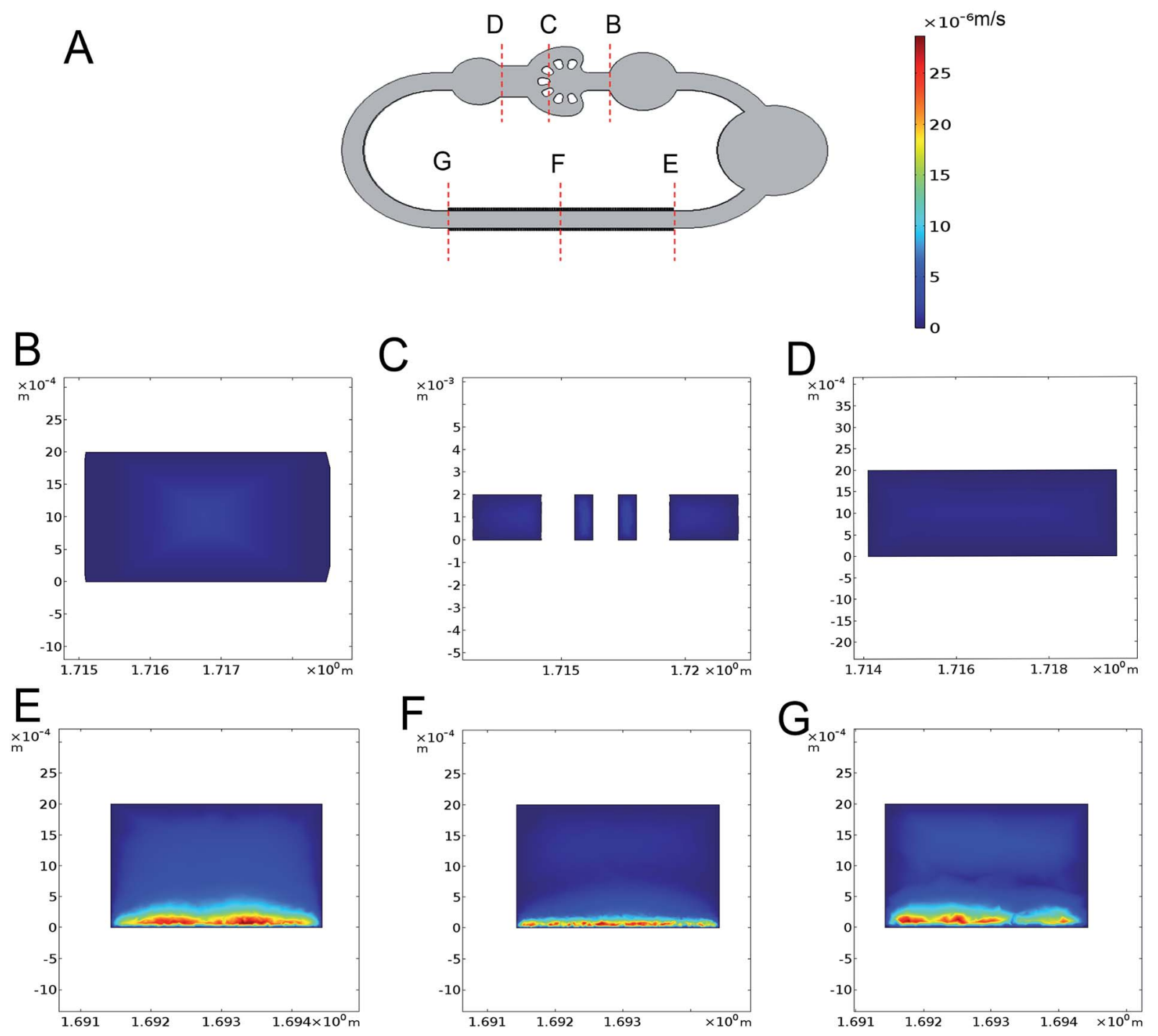

Fig. 3 3D simulation results for velocity distribution at $V_{p}=5 \mathrm{~V}, \sigma=1.43 \mathrm{~S} \mathrm{~m}^{-1}, f=1 \mathrm{MHz}$. (A) Vertical view of the self-circulating system and the velocity colour lengend. (B-G) Velocity distribution in different sections according to (A).

\subsection{Dynamic culturing and measurement of the parameters of fluid flow}

After a period of static culture, we use conductive copper adhesive tape to connect the self-circulating chip with the anode and the cathode of a signal generator (RIGOL, DG1022Z). We analysed the conductivity of the culture medium for $0 \mathrm{~h}, 12 \mathrm{~h}, 36 \mathrm{~h}$ and $84 \mathrm{~h}$ with a commercial electrical conductivity testing instrument (JY-TDS230) separately. The AC peak voltages of $2.5 \mathrm{~V}, 3 \mathrm{~V}, 3.5 \mathrm{~V}, 4 \mathrm{~V}, 4.5 \mathrm{~V}, 5 \mathrm{~V}$, $5.5 \mathrm{~V}$ and $6 \mathrm{~V}$, with a frequency of $10 \mathrm{MHz}$ and $1 \mathrm{MHz}$ are applied to the self-circulating chip. Polystyrene microspheres (Tianjin Baseline Chromtech Research Centre, diameter: 5 $\mu \mathrm{m}$, green) were resuspended in the solution and the time to pass a fixed distance was recorded to calculate the velocities of the medium. The temperature variation near the cell culture chamber is recorded with a commercial thermocouple temperature sensor (Zhaoxin, TM-902C) in real time.
AC peak voltages of $2.5 \mathrm{~V}, 3 \mathrm{~V}, 3.5 \mathrm{~V}, 4 \mathrm{~V}, 4.5 \mathrm{~V}, 5 \mathrm{~V}, 5.5 \mathrm{~V}, 6 \mathrm{~V}$, $10 \mathrm{~V}, 15 \mathrm{~V}$ and $20 \mathrm{~V}$ with frequency of $10 \mathrm{MHz}$ are applied to energize the chip separately and the entire measurement is accomplished in $37^{\circ} \mathrm{C}$ incubator for each voltage applied for $10 \mathrm{~min}$.

\subsection{Assessment of functional specificity of liver lobule}

Albumin is a specific secretion of the liver, and via ammonia metabolism, urea is also generated specifically by hepatocytes. By analysing the amount of these two products, the degree of functional normality can be determined. ${ }^{40}$ The albumin and urea were analysed on at 1.5, 2.5 and $3.5 \mathrm{~d}$ in static and dynamic culture environments, specifically. Albumin secretion was measured with a Human Serum albumin (ALB) ELISA Kit (Wuhan Chundu Biological Technology Co., Ltd.) according to standard instruction. The urea concentration was assayed with the Urea Assay Kit (Abcam, ab83362) with a NanoDrop 2000c 
(Thermo Scientific) according to the manufacturer's instructions.

\subsection{APAP treatment of liver lobule}

In our study, we selected the acetaminophen (APAP) (Abcam, ab120990) at different concentrations (0 (control), $10 \mathrm{mM}$ and $20 \mathrm{mM}$ ) to evaluate our liver lobule model. After dynamic culture in an incubator for $1.5,2.5$ or $3.5 \mathrm{~d}$, cell viability, urea synthesis and albumin secretion were evaluated from collected samples was taken out.

\subsection{Cell viability}

The Live/Dead Cell Imaging Kit (Life Technologies Corporation) was employed to detect the viability of cells in the liver lobule model. The liver plate samples cultured for $1.5 \mathrm{~d}, 2.5 \mathrm{~d}$ or 3.5 $\mathrm{d}$ were removed and $15 \mu \mathrm{l}$ of phosphate buffered saline (PBS) (HyClone) was added to the liver plate for washing 3 times for $5 \mathrm{~min}$ each, a total of $15 \mathrm{~min}$. The Live/Dead Cell Imaging Kit was added on the chamber to stain the liver plate. After the reaction proceeded for $15 \mathrm{~min}$ at $37{ }^{\circ} \mathrm{C}$, the chamber was washed with PBS 3 times. Finally, live/dead cell images were obtained with an electron microscope (Leica, DMi8), and the percentages of living and dead cells were counted to evaluate the viability of the liver lobule. Additionally, an elastomer base was mixed at proportion of $1: 10$ to pour on the mould. A slightly larger oval polymethylmethacrylate (PMMA) sheet (1.5 mm thick) with two exhaust vents covered the mould. After degassing in a vacuum drying oven and curing at $85{ }^{\circ} \mathrm{C}$ for $30 \mathrm{~min}$, the circulating flow channel is drafted.

\section{Results}

\subsection{Construction of self-circulating system}

To enhance the pumping efficiency in a unit of space, the parameters of the self-circulating system should be optimized. In the beginning, we experimented with using lengths of A, B, C and $\mathrm{D}$ of 100, 100, 150 and $350 \mu \mathrm{m}$, respectively, not shown in this paper. As is shown in Fig. 1B, we designed the dimensions of the electrodes according to the optimal geometry ratio $(0.3: 1: 3: 3-6)^{35}$ such that the lengths of A, B, C and D are 20 , 20,100 and $150 \mu \mathrm{m}$, respectively. This accuracy has approached the limitation of the laser marking machine with the line width accuracy, which is approximately $20 \mu \mathrm{m}$, and the number of electrode pairs reached 120 pairs compared to the previous 50 pairs. Due to the variation of energy distribution uniformity of the laser ablation, the boundary of the electrode area presents a zigzag appearance which could enhance the relative line boundary. The image of the electrode array is shown in Fig. 1C. And on the opposite of the electrode area, we arranged the cell culture area composed of buffer pools and cell-laden collagen area (Fig. 1D).

\subsection{Quantitative study of characteristic parameters of the self-circulating system}

The variation of electrical conductivity directly affects the state of ACET flow, particularly the velocity of fluid flow. And the electrical conductivities measured from the culture medium at 0, 1.5 and $3.5 \mathrm{~d}$ have been measured and shown in Fig. 2A. The measured electrical conductivity of the culture medium which contains 40\% DMEM and 40\% RPMI supplemented with $1 \% \mathrm{P} / \mathrm{S}$ and $10 \%$ FBS shows an increasing trend from 1.12 to $1.43 \mathrm{~S} \mathrm{~m}^{-1}$ for $3.5 \mathrm{~d}$. The reason is that with the extension of culture time, the cell supernatant ion concentration level was changing in varying degrees.

To quantitatively calculate the influence of voltage and frequency on the flow velocity and temperature of the cell culture channel, we selected the finite element analysis software (COMSOL Multiphysics 5.3) to observe dynamic change. In Fig. 2B and C, we simulated the flow velocity $1 \mathrm{~mm}$ above the bottom at the electrical conductivity of 1.12, 1.43 and $3.5 \mathrm{~S} \mathrm{~m}^{-1}$ to represent the condition of fresh, $3.5 \mathrm{~d}$ culture medium and maximum conductivity. The range of applied voltage was 2$5.5 \mathrm{~V}$ with the interval of $0.5 \mathrm{~V}$ and the applied frequency was 1 $\mathrm{MHz}$ and $10 \mathrm{MHz}$. The maximum velocity of $2.47 \mu \mathrm{m} \mathrm{s}^{-1}$ appeared at the electrical conductivity of $1.43 \mathrm{~S} \mathrm{~m}^{-1}$, the applied frequency of $10 \mathrm{MHz}$ and the voltage of $5.5 \mathrm{~V}$. The simulation curve obtained with the numerical simulation shows that under low electrical conductivity and low voltage, and the frequency variation did not have obvious influence on the velocity especially under $5 \mathrm{~V}$ (Fig. 2B and C). The experimental data (Fig. 2B and C), shows that the velocity was positively correlated with the level of frequency, electrical conductivity and applied voltage. From an overall perspective, the velocity from empirical observations was higher than the simulation calculation particularly at a low voltage and the exception appeared at high velocity. The highest velocity of $2.13 \mu \mathrm{m} \mathrm{s}^{-1}$ occurred at the electrical conductivity of $1.43 \mathrm{~S} \mathrm{~m}^{-1}$, applied frequency of $10 \mathrm{MHz}$ and voltage of $5.5 \mathrm{~V}$. To estimate the velocity in each diverging channel, we calculated the proportional relationship between the branch channel velocity (Fig. 2D) and the main flow velocity by simulating the 3D velocity of the total chip. At a voltage of $5 \mathrm{~V}$, frequency of $10 \mathrm{MHz}$ and electrical conductivity of $1.12 \mathrm{~S} \mathrm{~m}^{-1}$, the simulated flow of each channel was 20.3, 29.5, 53.8 and 96.1 $\mu \mathrm{l}$ per day, and the actual flow of each channel we evaluated are $17,25.8,47.4$ and $84 \mu \mathrm{l} \mathrm{d}^{-1}$, which fit the demand of $10 \mu \mathrm{l} \mathrm{d}^{-1}$ from our previous work. ${ }^{25}$ As shown in Fig. 3A-G, we simulated the velocities at the voltage of the $5 \mathrm{~V}$, frequency of $1 \mathrm{MHz}$ and electrical conductivity of $1.43 \mathrm{~S} \mathrm{~m}^{-1}$ and temperature variations appear mainly above electrode area.

Another critical factor is the cell damage generated by the AC electrothermal flow which cannot be neglected. To validate the extra input not affecting the cell culture, we use the finite element analysis software to simulate the $3 \mathrm{D}$ temperature distribution of the self-circulating system. From the result of the simulation at the applied voltage of $20 \mathrm{~V}$, frequency of $10 \mathrm{MHz}$ and the electrical conductivity of $1.43 \mathrm{~S} \mathrm{~m}^{-1}$, we found that the rise in temperature only clearly exist on the electrode pumping area (Fig. 4A-G). This is somewhat different from the actual situation that the rise in temperature is inevitable. This is because that we set the temperature boundary condition of the flow channel at $37^{\circ} \mathrm{C}$, whereas the fluid flow encapsulated in the PDMS channel which has low thermal conductivity would facilitate temperature accumulation. From the actual 


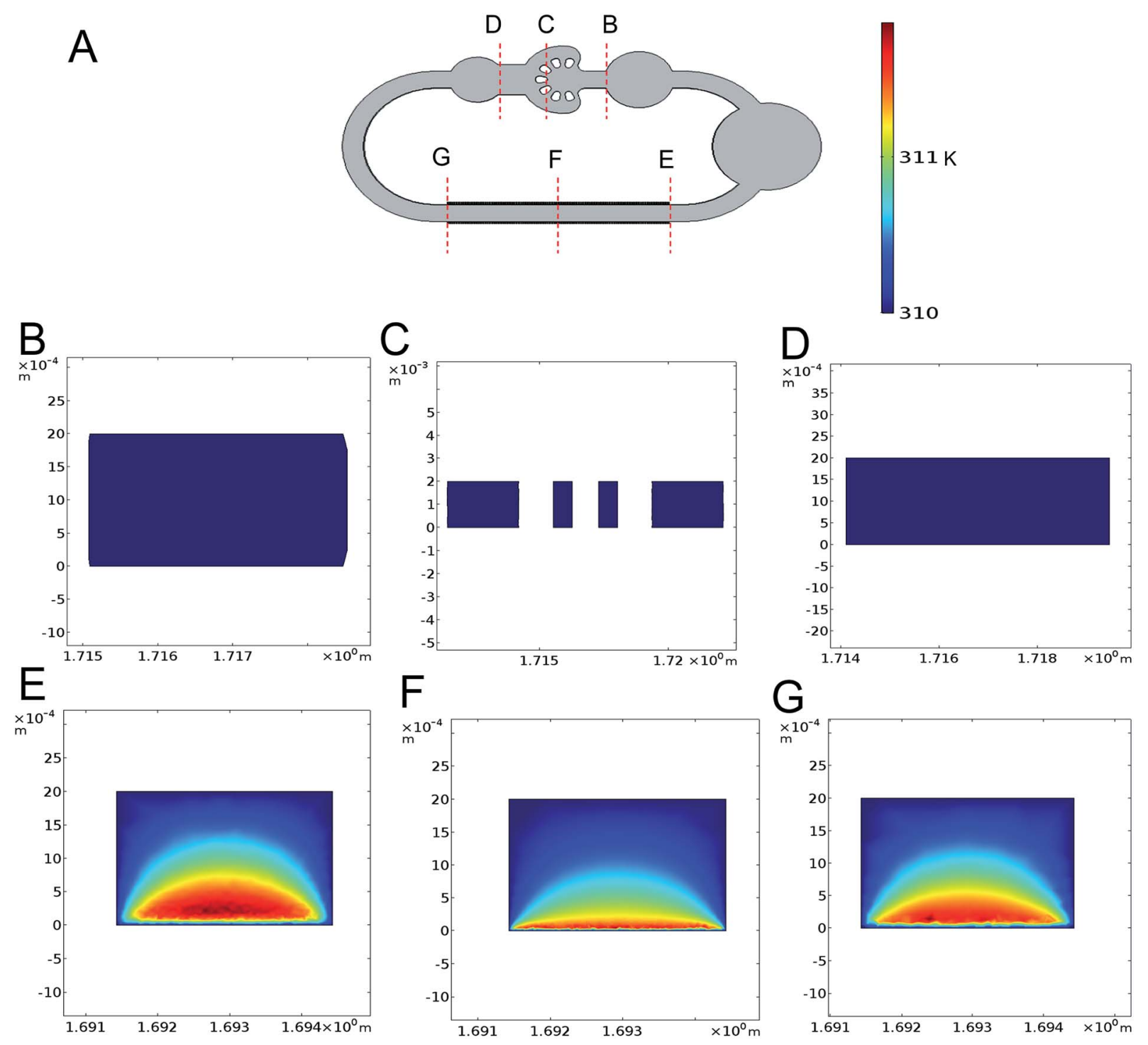

Fig. 4 3D simulation results for temperature distribution at $V_{p}=5 \mathrm{~V}, \sigma=1.43 \mathrm{~S} \mathrm{~m}^{-1}, f=10 \mathrm{MHz}$. (A) Vertical view of the self-circulating system and the temperature colour lengend. (B-G) Temperature distribution in different sections according to (A).

measurement of the temperature rise in cell culture area at applied voltage of $0-20 \mathrm{~V}$ with fresh culture medium, we found that there is no conspicuous rise in temperature under the voltage of $7 \mathrm{~V}$ with the accuracy of $0.1{ }^{\circ} \mathrm{C}$ (ESI Fig. S1†). And the highest temperature is no more than $1^{\circ} \mathrm{C}$ above the temperature in the incubator during the entire loading process.

\subsection{In vitro reconstruction of $3 \mathrm{D}$ radial shaped liver lobule model}

To achieve a 3D radial shaped liver lobule model integrating human liver cells and umbilical vein endothelial cells, the hierarchical approach of patterning was applied. The liver lobule model consists of three parts, i.e., the liver plate, HUVECs layer and flow channel. To construct the liver plate of the liver lobule, the hepatic cell-laden collagen was paved into the circular groove with some micro-pillars that efficiently fix the collagen efficiently (Fig. 5A). HUVECs were placed on the liver plate after positioning of the collagen such that it adheres tightly to the surface of the collagen (Fig. 5A). The radial shaped patterning of HUVECs was guided by the corresponding radial shaped channel that the branches of the liver lobule model can be regarded as an independent test unit. And referring to our past research, the density of cell-laden collagen was set as $1.5 \times 10^{4} \mathrm{ml}^{-1}$, which is considered as suitable for cell growth.

To observe the multilayer structure in detail, the liver plate accompanied by HUVECs was imaged and laminated by confocal microscopy. Compared with growth on the collagen by gravity, the HUVECS layer was easily adhered to the surface of PDMS. However, after $12 \mathrm{~h}$ of static culture in the incubator, a radial pattern of HUVECs was formed on the surface of the fan-shaped liver plate (Fig. 5C-E). And due to the poor mechanical properties of the cell-laden collagen, we tested the flow effect of a that a maximum flow velocity of $20 \mu \mathrm{m} \mathrm{s}^{-1}$ from the static state to dynamic state and found that it did not damage the morphology of the liver plate. 
A 1

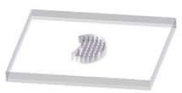

4

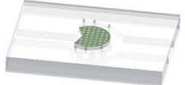

2

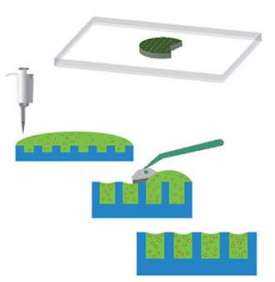

5

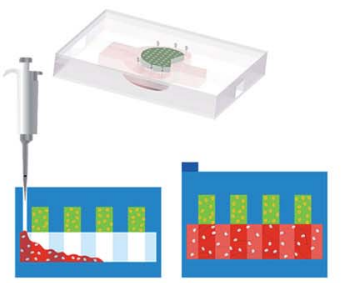

3

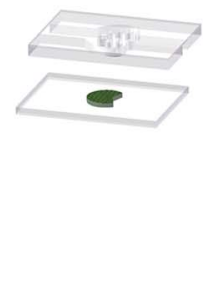

6

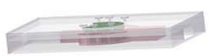

B

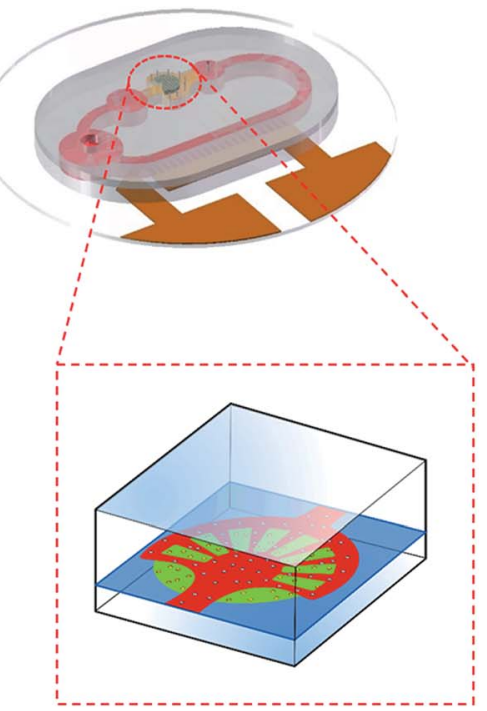

C

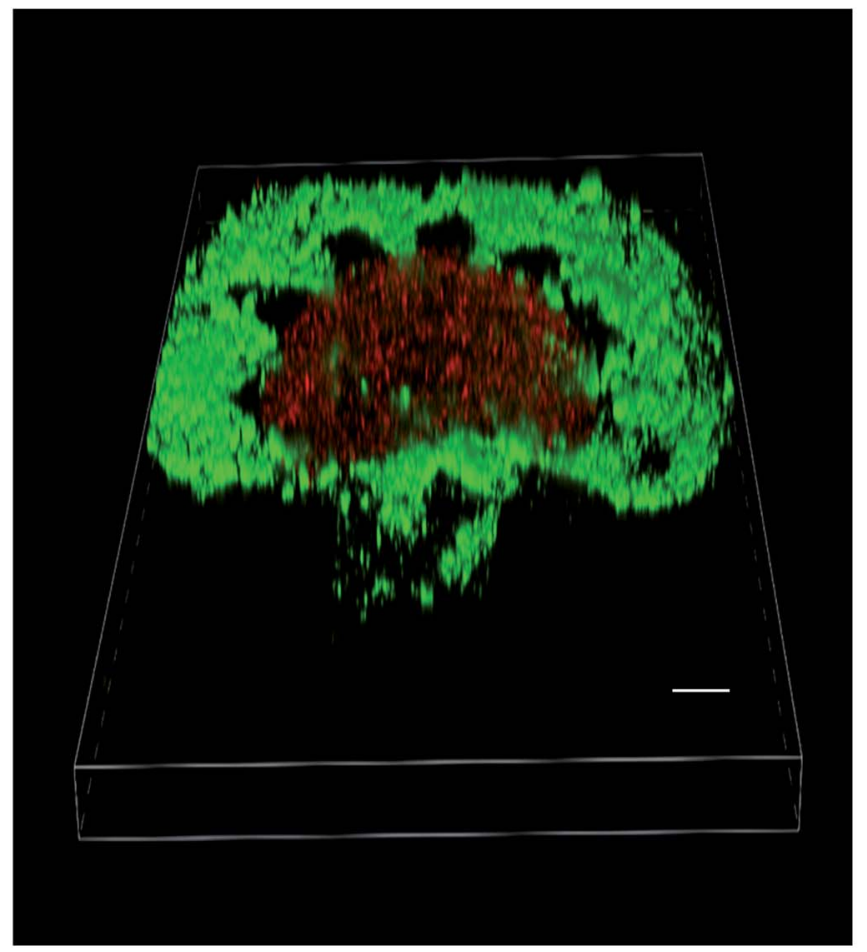

D

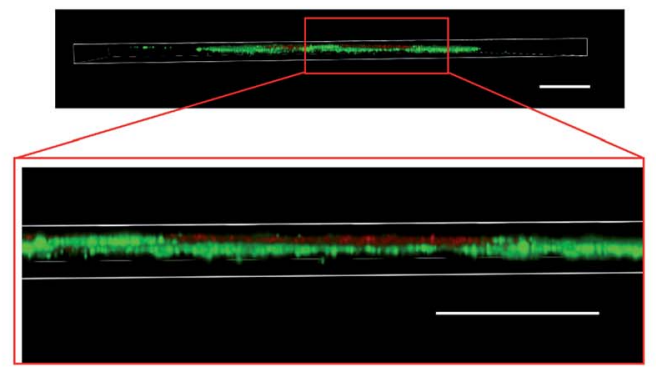

E

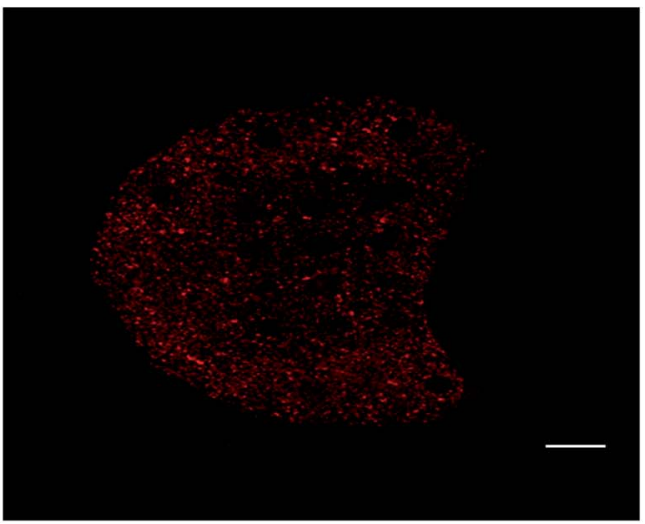

Fig. 5 3D biomimetic liver-lobule model. (A) Fabrication process of the liver-lobule model. (B) Schematic graph of the self-circulating system with the radial multi-layer liver-lobule model. (C) Complete view of the liver-lobule model (the upper one is the liver plate, and the lower one is the endothelial layer), scale bar $=1.5 \mathrm{~mm}$. (D) Confocal side view of the 3D liver-lobule model, scale bar $=1 \mathrm{~mm}$. (E) Confocal top view of the liver plate, scale bar $=1 \mathrm{~mm}$.

\subsection{Quantitative analysis of liver lobule model in dynamic} culture and static culture

To better understand the activities of liver-lobule model in different situations, the cell viability of the $3 \mathrm{D}$ liver lobule model in a dynamic environment and static environment were assessed. We apply a voltage of $5 \mathrm{~V}$ to generate a velocity of approximately $2 \mu \mathrm{m} \mathrm{s}^{-1}$ in the main flow channel. And after a $12 \mathrm{~h}$ static culture followed another 1,2 and $3 \mathrm{~d}$ of continuous dynamic culturing, we calculate the ratio of live cells to dead cells. The images (Fig. 6A) clearly show the state of cell proliferation and dead. Cell viability was negatively correlated with 

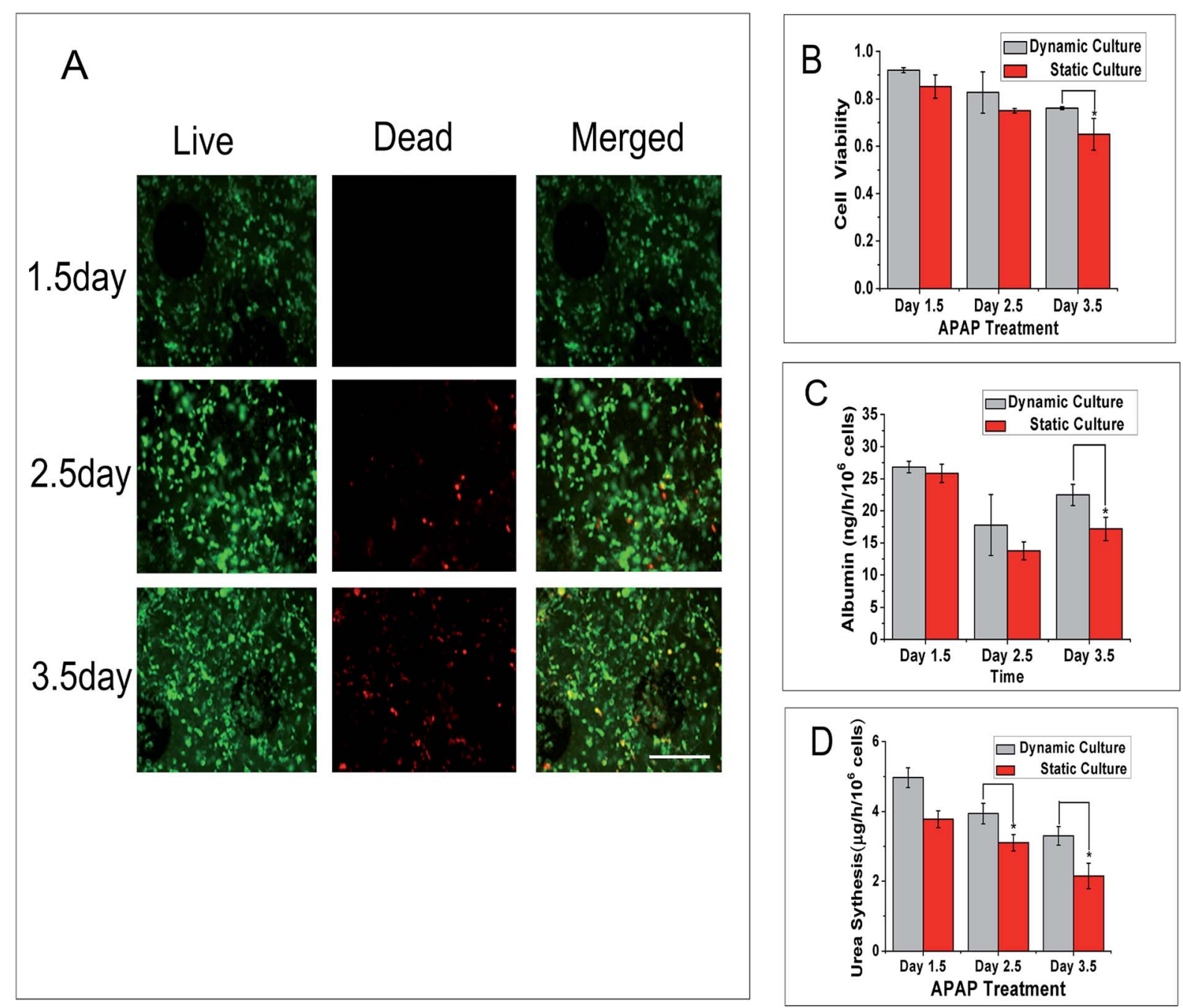

Fig. 6 Viability and characteristic representative of the liver-lobule model under dynamic culture and static culture for 1.5, 2.5, and 3.5 d. (A) Live/ dead image of the liver lobule model under dynamic culture for 1.5, 2.5, and $3.5 \mathrm{~d}$. The live cells were marked green, and the dead cells were marked red, scale bar $=500 \mu \mathrm{m}$. (B) Cell viability of the liver lobule model under different culture conditions over time. (C) Albumin secretion level of the liver-lobule model under different culture conditions over time. (D) Urea production level of the liver lobule model under different culture conditions over time, the error bars represent the standard deviations calculated from separate assays $(n=3),(* p<0.05)$.

the culture time. Through the quantitative comparison of cell viability in dynamic and static culture (Fig. 6B), we found that cells in dynamic culture have a higher viability than in static culture. The viability of cells in dynamic culture maintained a ratio of above $80 \%$ for $2.5 \mathrm{~d}$ and above $75 \%$ for $3.5 \mathrm{~d}$ that is suitable for short-term training.

Albumin secretion is a main liver-specific biomarker that can be used to evaluate the activity of hepatocytes and urea metabolism is also regarded as a major measurement of hepatocyte functionality. In our research, we measured the content of human albumin by ELISA to quantitatively anabase the metabolic rate. The chart (Fig. 6C) shows that the albumin secretion was not linearly related to time. The secretion of albumin in dynamic culture in these three days are approximately 27,18 and $22 \mathrm{ng}$ per h per $10^{6}$ cells, respectively, and 26 , 14 and $17 \mathrm{ng}$ per h per $10^{6}$ cells in static culture. Therefore the liver lobule model cultured in a dynamic environment have a higher albumin synthesis level than that in static culture. As shown in another set of data (Fig. 6D), we used the Urea Assay Kit to quantitatively analyse the ammonia metabolism of our liver lobule model. The urea production diminished over time and the trend is the same as cell viability change over time. The level of urea metabolism in dynamic culture is approximately $0.8-1.2 \mu \mathrm{g}$ per $\mathrm{h}$ per $10^{6}$ cells more than in static culture.

\subsection{APAP drug reaction to self-circulating liver lobule model}

Liver, the biggest interior organ in our body, plays a crucial role in the metabolism and detoxification for drugs. Hence, whether the self-circulating liver lobule model can be applied to screen for drug reactions is a key for our model to simulate the function of the liver in vivo. We selected the hepatotoxic drug acetaminophen at different concentrations of 0,10 and $20 \mathrm{mM}$. We cultured the liver lobule model in a static environment for $12 \mathrm{~h}$ followed 1, 2 and $3 \mathrm{~d}$ dynamic culture at an AC voltage of $5 \mathrm{~V}$ and frequency of $10 \mathrm{MHz}$. 

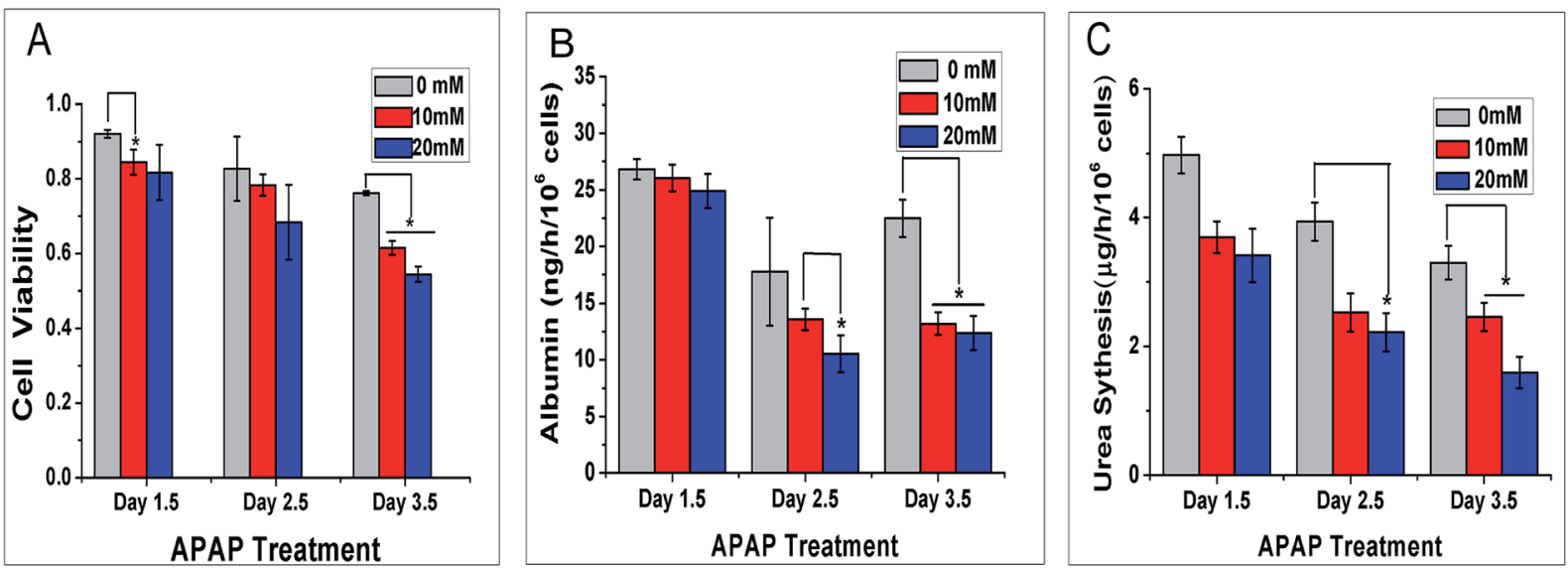

Fig. 7 Viability and liver-specific functions of the liver-lobule model under ACET dynamic culture with different APAP treatments for 1.5, 2.5, and 3.5 days. (A) Cell viability of the liver-lobule model at different concentrations of APAP agent over time. (B) Albumin secretion level of the liverlobule model at different concentrations of APAP agent over time. (C) Urea production level of the liver-lobule model at different concentrations of APAP agent over time, the error bars represent the standard deviations calculated from separate assays $(n=3),\left({ }^{*} p<0.05\right)$.

The statistical results of cell viability are shown in Fig. 7A. From the histogram, under APAP treatment, cell viability is negatively correlated with the concentration of APAP. When the concentration of APAP was $10 \mathrm{mM}$ and $20 \mathrm{mM}$, cell viability was significantly reduced with time compared to the control group.

The albumin secretion level of the self-circulating liver lobule model is shown in Fig. 7B. Similar to the previous static/ dynamic comparison, the albumin secretion increases and decreases, which may be caused by the increase of the basic number and secretory activity changes. The albumin secretion at APAP concentration of $10 \mathrm{mM}$ and $20 \mathrm{mM}$ declined sharply at 2.5 and $3.5 \mathrm{~d}$ which reflects the inhibition of cell activity. As the energy is removed to support other functions to maintain basal metabolism, albumin secretion, a relatively high level metabolic product is reduced.

Urea production by APAP dynamic treatment at different concentrations for $1.5,2.5$ and $3.5 \mathrm{~d}$ were also evaluated by the method above. Different from the data collected for the cell viability and albumin secretion, the secretions of urea decreased in the first two days of dynamic culture, particularly at the concentration of $20 \mathrm{mM}$ (Fig. 7C). This result is mainly caused by mitochondria damaged by APAP to varying degrees, which directly affects ammonia metabolism. At $3.5 \mathrm{~d}$, the residual citrulline that maintained in the mitochondria was released, with cell organelle break down that can sustain urea production at a certain level. Based on these results, the effect of medicinal reactions on liver at low concentration has demonstrated that liver lobule model was injured in this selfcirculating system obviously. For the current situation of no self-recirculating drug test model, our model can be used to detect drug metabolism in the liver under a dynamic environment.

\section{Discussion}

Based on our previous research, we constructed a selfcirculating system on a chip integrating a 3D liver lobule model that can be used for dynamic drug screening and pathological study. The innovation of our research covered in this letter include the following: (1) we use a laser etching process to fabricate interdigitated electrodes on ITO that is rapid, more efficient and more inexpensive than the lift-off process of ITO or platinum..$^{41-43}$ The minimum width of the electrode with the etching process can be controlled is $20 \mu \mathrm{m}$, whereas wet etching reaches approximately $50 \mu \mathrm{m}$, requiring a relatively high level of control for etching time and prior pattern processing. ${ }^{28,42,44}$ The result is that we increase the number of electrodes in a limited space approximately twice that used in the etching process. (2) Our self-circulating system can produce interstitial flow and vascular flow depending on the input parameters. The system integrated relatively more complex flow channels, and through actual measurement combined with simulation by finite element analysis, we achieved control of fluid velocity in branches at different applied $\mathrm{AC}$ voltages and frequencies without any thermal damage to the cells (as indicated by viability studies). Additionally, different from an AC electrothermal flow-driven microfluidic chip in which fluid affects only the cells on the electrode side,$^{28}$ cells can be seeded on both sides of the channel by a method of double-sided bonding of the flow channel, which has scalable potential. (3) We constructed a membrane-free biomimetic liver lobule model, which form as a real multilayer structure of different types of cells in a spatial dimension. It is well-known that the co-culture of HepG2 with other types of specific cells would facilitate the bionics of liver function ${ }^{45-47}$ and the technology of cell-patterning can be further applied in this area ${ }^{48}$ Through different methods, such as 3D stereolithography bioprinting, ${ }^{21}$ enhanced field-induced dielectrophoresis (DEP) ${ }^{20}$ or patterned collagen adsorbing cells ${ }^{24}$ a radial shaped liver lobule model can be achieved. However, HUVECs and HepG2 cells are seeded on different interfaces, and blood does not contact HepG2 cells directly in an actual 3D biomimetic liver lobule. In addition, another method is the multilayer co-cultivation, which requires the porous membranes, such as PET or silicon nitride, ${ }^{17-19}$ and has 
a poor permeability that is not as good as direct contact at the bionic level. The remaining methods such as freestanding stacked mesh-like hydrogel sheets that produce a direct contact multilayer structure with an alginate precursor and calcium chloride dihydrate would not be used as a method for long-term culture due to the poor biocompatibility and expandability of cells. $^{22}$ Our 3D biomimetic liver lobule model is a sandwich structure in which the endothelial layer is tightly attached to underlying hepatic cell-laden layer, which can protect hepatocytes from the destructive effect of fluid shear stress while ensuing effective nutrient transmission. ${ }^{5,23}$ By comparison, in dynamic culture and static culture, we observed that the flow shear force would enhance the metabolism of cells and from additional experiments in dynamic culture, APAP treatment at different concentration confirmed the feasibility of pharmacokinetic research. The results of some routine testing indicators such as cell viability, albumin secretion and urea synthesis conformed to general experimental laws.

However, there remain some deficiencies in our research. (1) Due to the flow channel requiring a certain height to generate an electrothermal vortex to actuate the fluid, the size of the entire chip is larger than a traditional microfluidic chip. Therefore, optimizing the entire structural design is required in order to reduce the resistance of fluid flow and improve the utilization of space. (2) The current development of organ-onchip is to integrate different organs in a parallel system. Therefore, the next step is to co-cultivate multiple organs in a closed-loop system and verify the interaction between different tissues and organs to construct a relatively complete metabolic system.

\section{Conclusions}

In summary, we fabricated an AC electrothermal self-circulating system by laser etching on ITO glass that can easily and efficiently improve the process of patterning of inter-digital electrodes. A 3D biomimetic liver lobule model shaped similar to a sandwich with hepatocytes on the bottom layer and an endothelium layer in the middle- was integrated with the fluid channel. By adjusting the input signal, the fluid velocity in the flow channel can be controlled without complex external devices, and temperature increases are rarely observed at the applied voltage of $7 \mathrm{~V}$ and frequency of $10 \mathrm{MHz}$. By comparing the metabolism of liver lobule in static and dynamic culture, we verified that the stress induced by the flow is more suitable for the growth of our liver lobule. In addition, we constructed a hepatic drug test model using APAP treatment at different concentrations in the dynamic culture. The results of cell viability, albumin secretion and urea synthesis showed that our self-circulating liver lobule system has the pharmacokinetic properties of the liver. Our future work will focus on the research and development of materials to reduce the resistance of fluid flow and additionally decrease the size of the system. We also plan to integrate the self-circulating system with more tissue or organ types to achieve a true sense of a self-circulating human model on a chip.

\section{Conflicts of interest}

There are no conflicts to declare.

\section{Acknowledgements}

This study was kindly supported by the Projects of Basic Research of Shenzhen, China (No. JCYJ20170817162507554, JCYJ20170817162121275， JCYJ20170817162629875， \& JCYJ20170412101508433).

\section{References}

1 D. Huh, H. J. Kim, J. P. Fraser, D. E. Shea, M. Khan, A. Bahinski, G. A. Hamilton and D. E. Ingber, Nat. Protoc., 2013, 8, 2135-2157.

2 S. R. Khetani and S. N. Bhatia, Nat. Biotechnol., 2008, 26, 120126.

3 D. Huh, G. A. Hamilton and D. E. Ingber, Trends Cell Biol., 2011, 21, 745.

4 A. Skardal, T. Shupe and A. Atala, Drug Discovery Today, 2016, 21, 1399-1411.

5 Y. Wang, W. Su, L. Wang, L. Jiang, Y. Liu, L. Hui and J. Qin, Toxicol. Res., 2017, 7, 13-21.

6 Z. Li, L. Jiang, Y. Zhu, W. Su, C. Xu, T. Tao, Y. Shi and J. Qin, Toxicol. In Vitro, 2018, 46.

7 S. A. Lee, D. Y. No, E. Kang, J. Ju, D. S. Kim and S. H. Lee, Lab Chip, 2013, 13, 3529-3537.

8 J. M. Prot, C. Aninat, L. Griscom, F. Razan, C. Brochot, C. G. Guillouzo, C. Legallais, A. Corlu and E. Leclerc, Biotechnol. Bioeng., 2011, 108, 1704-1715.

9 L. A. Vernetti, N. Senutovitch, R. Boltz, R. Debiasio, Y. S. Tong, A. Gough and D. L. Taylor, Exp. Biol. Med., 2016, 241, 101.

10 C. T. Ho, R. Z. Lin, W. Y. Chang, H. Y. Chang and C. H. Liu, Lab Chip, 2006, 6, 724-734.

11 Y. B. Kang, T. R. Sodunke, J. Lamontagne, J. Cirillo, C. Rajiv, M. J. Bouchard and M. Noh, Biotechnol. Bioeng., 2015, 112, 2571-2582.

12 L. Prodanov, R. Jindal, S. S. Bale, M. Hegde, W. J. Mccarty, I. Golberg, A. Bhushan, M. L. Yarmush and O. B. Usta, Biotechnol. Bioeng., 2016, 113, 241-246.

13 Y. Du, N. Li, H. Yang, C. Luo, Y. Gong, C. Tong, Y. Gao, S. Lü and M. Long, Lab Chip, 2017, 17, 782-794.

14 S. Zhang, L. Xia, C. H. Kang, G. Xiao, S. M. Ong, Y. C. Toh, H. L. Leo, N. D. Van, S. H. Kan and H. H. Tang, Biomaterials, 2008, 29, 3993-4002.

15 C. T. Ho, R. Z. Lin, R. J. Chen, C. K. Chin, S. E. Gong, H. Y. Chang, H. L. Peng, L. Hsu, T. R. Yew and S. F. Chang, Lab Chip, 2013, 13, 3578-3587.

16 X. Ma, X. Qu, W. Zhu, Y. S. Li, S. Yuan, H. Zhang, J. Liu, P. Wang, C. S. Lai and F. Zanella, Proc. Natl. Acad. Sci. U. S. A., 2016, 113, 2206.

17 J. Son, C. Y. Bae and J. K. Park, Biotechnol. J., 2016, 11, 585591.

18 A. A. Banaeiyan, J. Theobald, J. Paukštyte, S. Wölfl, C. B. Adiels and M. Goksör, Biofabrication, 2017, 9, 15014. 
19 M. Chao, Z. Lei, E. M. Zhou, J. Xu, S. Shen and J. Wang, Anal. Chem., 2016, 88, 1719.

20 S. Mi, X. Yi, Z. Du, Y. Xu and W. Sun, Biofabrication, 2018, 10, 25010

21 X. Liu, K. Yang, A. Wadhwa, S. Eda, S. Li and J. Wu, Sens. Actuators, A, 2011, 171, 406-413.

22 Y. Quan and W. Jie, Biomed. Microdevices, 2013, 15, 125-133.

23 Q. Lang, Y. Wu, Y. Ren, Y. Tao, L. Lei and H. Jiang, ACS Appl. Mater. Interfaces, 2015, 7, 26792-26801.

24 R. H. Vafaie, H. B. Ghavifekr, H. Lintel, J. Brugger and P. Renaud, Electrophoresis, 2016, 37, 719-726.

25 W. Y. Ng, S. Goh, Y. C. Lam, C. Yang and I. Rodríguez, Lab Chip, 2009, 9, 802-809.

26 N. G. Green, A. Ramos, A. Gonzalez, H. Morgan and A. Castellanos, Phys. Rev. E: Stat. Phys., Plasmas, Fluids, Relat. Interdiscip. Top., 2000, 61, 4011.

27 A. Castellanos, A. Ramos, A. González, N. G. Green and H. Morgan, J. Phys. D: Appl. Phys., 2003, 36, 2584-2597.

28 A. Ramos, H. Morgan, N. G. Green and A. Castellanos, J. Phys. D: Appl. Phys., 1998, 31, 2338-2353.

29 M. Lian and J. Wu, Microfluid. Nanofluid., 2009, 7, 757.

30 Q. Yuan, K. Yang and J. Wu, Microfluid. Nanofluid., 2014, 16, 167-178.

31 A. González, A. Ramos, H. Morgan, N. G. Green and A. Castellanos, J. Fluid Mech., 2006, 564, 415-433.

32 F. Gao, S. S. Naini, J. Wagner and R. Miller, Int. J. Refrig., 2016, 73, 99-110.

33 F. Gao, L. Zhao, M. C. Boufadel, T. King, B. Robinson, R. Conmy and R. Miller, Appl. Ocean Res., 2017, 68, 77-90.

34 J. Wu, M. Lian and K. Yang, Appl. Phys. Lett., 2007, 90, 56302. 35 R. Riahi, S. A. M. Shaegh, M. Ghaderi, S. Z. Yu, R. S. Su, J. Aleman, S. Massa, D. Kim, M. R. Dokmeci and A. Khademhosseini, Sci. Rep., 2016, 6, 24598.

36 A. Salari, M. Navi and C. Dalton, Biomicrofluidics, 2015, 9, 14113.
37 H. C. Feldman, M. Sigurdson and C. D. Meinhart, Lab Chip, 2007, 7, 1553-1559.

38 I. Wagner, E. M. Materne, S. Brincker, U. Süssbier, C. Frädrich, M. Busek, F. Sonntag, D. A. Sakharov, E. V. Trushkin and A. G. Tonevitsky, Lab Chip, 2013, 13, 3538-3547.

39 W. Y. Ng, S. Goh, Y. C. Lam, C. Yang and I. Rodríguez, Lab Chip, 2009, 9, 802-809.

40 B. J. Kane, M. J. Zinner, M. L. Yarmush and M. Toner, Anal. Chem., 2006, 78, 4291.

41 Y. Du, N. Li, H. Yang, C. Luo, Y. Gong, C. Tong, Y. Gao, S. Lü and M. Long, Lab Chip, 2017, 17, 782-794.

42 J. M. Prot, C. Aninat, L. Griscom, F. Razan, C. Brochot, C. G. Guillouzo, C. Legallais, A. Corlu and E. Leclerc, Biotechnol. Bioeng., 2011, 108, 1704-1715.

43 B. J. Kane, M. J. Zinner, M. L. Yarmush and M. Toner, Anal. Chem., 2006, 78, 4291.

44 C. T. Ho, R. Z. Lin, R. J. Chen, et al., Liver-cell patterning lab chip: mimicking the morphology of liver lobule tissue, $L a b$ Chip, 2013, 13(18), 3578-3587.

45 N. W. Y. Wan, N. A. Kadri and F. Ibrahim, Cell Patterning for Liver Tissue Engineering via Dielectrophoretic Mechanisms, Sensors, 2014, 14(7), 11714-11734.

46 C. T. Ho, R. Z. Lin and H. Y. Chang, et al., In-Vitro Rapid Centimeter-Scale Reconstruction of Lobule-Mimetic Liver Tissue Employing Dielectrophoresis-Based Cell Patterning, Solid-State Sensors, Actuators and Microsystems Conference Transducers 2007, International, IEEE, 2007, pp. 351-354.

47 S. J. Kim, H. Ise, M. Goto, et al., Interactions of vimentin- or desmin-expressing liver cells with $\mathrm{N}$-acetylglucosaminebearing polymers, Biomaterials, 2012, 33(7), 2154-2164.

48 R. Chang, K. Emami, H. Wu, et al., Biofabrication of a threedimensional liver micro-organ as an in vitro drug metabolism model, Biofabrication, 2010, 2(4), 045004. 